\title{
Fuzzy Vehicle Control System for Single Intersection
}

\author{
Shashank Sahu, Rashi Agarwal, Rajesh Kumar Tyagi
}

\begin{abstract}
Traffic light at the intersection is an important part of the daily life of the urban cities. Traffic light needs to be managed properly so that a large number of vehicles able to cross the intersection. Current traffic light system is not able to allot efficient traffic light cycle length to vehicles. It is because of the traffic system is based on the fixed cycle length. Today traffic is highly fluctuating. Number of vehicles in the traffic varies with respect to time and day. To handle this situation, the paper proposes a vehicle control system using fuzzy logic: Fuzzy Vehicle Control System (FVCS). FVCS allots dynamic cycle length signal duration to vehicles based on the present traffic available at the intersection. Experiments have been performed on the proposed FVCS architecture using MATLAB. Experiments show that a reduction of $28.92 \%$ can be achieved in the number of vehicles that are waiting at the intersection to cross it.
\end{abstract}

Index Terms: Cycle Length, Green Light Time, Intersection Fuzzy Logic, Vehicle Control System.

\section{INTRODUCTION}

Number of vehicles coming at the intersection is of highly dynamic nature at the intersection today. Fixed time control traffic light signal at the intersection is not suitable to handle the dynamic nature of vehicles. It shows a need of automatic and dynamic traffic signal to provide a solution to the traffic jam at the intersection. Generally, traffic lights timings are set on basis of historical data. It can not control the traffic which is fluctuating. There should be a decision making procedure based on the current traffic pattern. The objective of traffic control management is to minimize delay so that the movement of vehicles can be maximized at the intersection [7], [8]. Vehicle control system should be able to handle the uncertainty of the number of vehicles arriving at the intersection. Establishing an effective control system at the intersection is also reduces pollution in the environment [9]. Fuzzy logic is able to handle the uncertainty in vehicle movement. The fuzzy system mechanism preserves uncertainty and infers duration of green light for smooth conduct of crossing vehicles at the intersection. Fuzzy system is able to take decision similar to human being. Major research is going on applications of intelligent techniques. These techniques may be like fuzzy logic, expert system genetic algorithm and neural networks. These techniques are also applicable to traffic control signals [1]-[6]. Driving behavior models based on mixed traffic have been presented

Revised Manuscript Received on July 5, 2019.

Shashank Sahu, Department of Computer Science \& Engineering, Research Scholar, Sharda University, Greater Noida and Associate Professor, Ajay Kumar Garg Engineering College Ghaziabad, India.

Rashi Agarwal, Department of Computer Applications, Galgotias College of Engineering \& Technology, Greater Noida, India.

Rajesh Kumar Tyagi, Department of Computer Science \& Engineering, Amity University, Gurugram, India. in [15]. The road traffic should be able to include complex flows such as consideration of pedestrians, road environment, and road traffic with multiple vehicles [16].

A fuzzy based traffic signal system may use sensors to collect data related to vehicle count. Accordingly, traffic system may take decision for the duration of traffic cycle length. The cycle length of the traffic system should be optimized according to the traffic presently exists at the intersection. Fuzzy logic is able to provide a controlling mechanism at the intersection which is suitable for heavy traffic [10]. Fuzzy logic has been applied first in traffic control in [11]. They provided a fuzzy logic system for a single intersection two phase one-way. A model is presented in [12] based on fuzzy logic to manage important personnel. Important personnel movement creates problems at the road that may lead to accidental cases. A model with a single junction that incorporates urgent vehicle management is presented in [14]. Vehicle control management models using fuzzy logic to handle heavy traffic have been presented in [17]-[20]. A model using fuzzy model that avoids obstacles automatically for car navigation is presented in [20]. In [21], green time duration is calculated dynamically using fuzzy control. Fuzzy logic can also be used in other area of applications related to vehicles [22]. Further paper is organized in four sections. Section II presents proposed work Fuzzy Vehicle Control System and describes details of the proposed model. It is followed by section III that discusses the experiment which has been conducted for the verification of the FVCS architecture. Section IV presents the results that have been obtained after the experiment. Conclusion of the paper is presented in the section $\mathrm{V}$.

\section{FUZZY VEHICLE CONTROL SYSTEM}

We propose architecture that allocates dynamic green cycle length to vehicles for crossing the intersection. The model is based on fuzzy logic. Fuzzy Vehicle Control System (FVCS) architecture considers a single two phase intersection as shown in Fig. 1. Two phases are North-South phase and East-West phase. North-South phase will be considered as Phase 1 (i.e. Phase A) and East-West phase will be considered as Phase 2 (i.e. Phase B) in the paper. When vehicles at phase 1 are moving then vehicles at phase 2 is stopped and waiting for crossing the intersection. Similarly, when vehicles at phase 2 are moving then vehicles at phase 1 are stopped and waiting for crossing the intersection. FVCS architecture is presented in Fig. 2. The architecture provides cycle length based on current traffic present at the intersection. 
The architecture counts the number of vehicles of both the phases (Phase $1 \&$ Phase 2) and accordingly calculates cycle length for the traffic lights. The architecture consists of five components which are described in the following subsections.

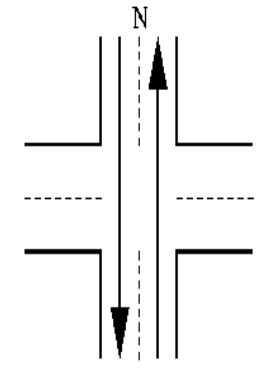

$\mathrm{S}$ Phase A

Fig. 1 Two Phase Intersection

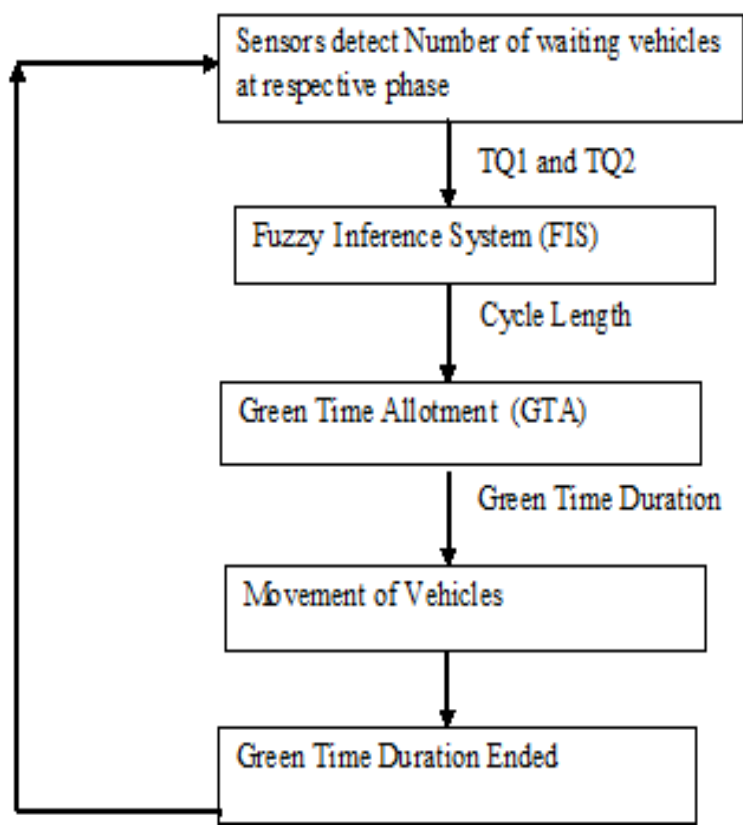

Fig. 2 Fuzzy Vehicle Control System (FVCS) Architecture

\section{A. Sensors to detect vehicles}

This component of FVCS detects the number of vehicles presently exists in both phases and they are waiting to cross the intersection. It uses two sensors (say S1 and S2) for each phase for finding the number of vehicles. First sensor (S1) is situated near to the traffic light and second sensor (S2) is situated 100 meters away from the first sensor. First sensor (S1) is to count vehicles passing from the intersection when the green light is on. Second sensor (S2) counts vehicles arriving towards the intersection. When the green light duration ends, difference between number of vehicles detected by sensor $\mathrm{S} 2$ and number of vehicles detected by sensor S1 denotes Traffic Queue (TQ) of a phase. Similarly, Traffic Queue (TQ) is determined for the other phase also. Traffic queue is also called waiting vehicles because it represents number of vehicles waiting for green light to be turn on so that they are able to cross the intersection. Equation (1) is showing the equation that determines Traffic Queue which is number of vehicles waiting at intersection for crossing it.

$$
\mathrm{TQ}=\text { count }(\mathrm{S} 2)-\operatorname{count}(\mathrm{S} 1)
$$

Here, TQ denotes number of vehicles of a phase waiting for crossing the intersection and the function count() returns number of vehicles which have been detected by a particular sensor. Here, it is assumed that rate of vehicles arriving towards the intersection is higher than rate of vehicles crossing the intersection. When the rate of vehicles crossing the intersection is higher than the rate of vehicles arriving towards the intersection then the absolute value of TQ is taken for further analysis.

After finding traffic quantity of both phases of the intersection, fuzzy inference system module is called. We are using two inputs for fuzzy inference system, one is Traffic Queue-1 (TQ1) which has been determined from phase 1 and other is Traffic Queue-2 (TQ2) which has been determined from phase 2 .

\section{B. Fuzzy Inference System}

Fuzzy Inference System (FIS) is responsible for producing cycle length on the basis of the current traffic. It uses Mamdani Fuzzy inference system. FIS has two input parameters: TQ1 and TQ2. Where TQ1 represents traffic queue from phase 1 and TQ2 represents traffic queue from phase 2. Output parameter of FIC is Cycle Length. Values of input parameters are in the range from 1 to 80 vehicles. It is assumed that 1.5 seconds are needed to pass single vehicle from the intersection. Accordingly, output parameter cycle length is designed which is of the maximum of 120 seconds for longest traffic queue of 80 vehicles. Linguistic variables of input parameters TQ1 and TQ2 are Very Small (VS), Small (S), Medium (M), Large (L), and Very Large (VL). There are 25 fuzzy rules which are designed in the fuzzy inference system (FIS). These rules provide suitable cycle length on the basis of currently available traffic for the intersection. We have designed 25 fuzzy rules suitably to cater various types of traffic queues as well as to reduce the complexity of the system. Increasing the number of fuzzy rules in the fuzzy system is also increasing the complexity of system [13]. Fig. 3 shows 25 fuzzy rules.

FIS uses triangular-shaped membership function to characterize its input and output variables. Fig. 4 to 6 show triangular membership functions of TQ1, TQ2 and Cycle Length and Fig. 7 shows surface area of FIS. All members' functions are uniformly distributed. The crisp value of fuzzy set is determined by the defuzzification process. The defuzzification process finalizes one value from the resultant fuzzy set. There are various methods for the defuzzification. In our proposed architecture, we use SOM (smallest of maximum) method to determine crisp value of fuzzy set.

\section{Green Time Allotment (GTA)}

Cycle length which is provided by FVCS is then further divided into suitable green light timings for both phases of the intersection. Green light timing is calculated in the proportion of the number of vehicles currently present in the respective phase. If a phase is having small number of vehicles then accordingly small green time duration is allocated from the cycle length. 
If a phase is having high number of vehicles then large green time duration is allocated from the cycle length. Allocation of green time duration for the respective phase from the cycle length is calculated according to percentage of vehicles currently available in the respective phase. Equation (1) \& (2) are used to calculate green light time duration. GT1 denotes green light time duration of phase 1 and GT2 denotes green light time duration of phase 2. CL denotes cycle length which is provided by the FVCS.

$$
\begin{aligned}
& \mathrm{GT} 1=(\mathrm{TQ} 1 /(\mathrm{TQ} 1+\mathrm{TQ} 2)) * \mathrm{CL} \\
& \mathrm{GT} 2=(\mathrm{TQ} 2 /(\mathrm{TQ} 1+\mathrm{TQ} 2)) * \mathrm{CL}
\end{aligned}
$$

GT1 and GT2 are allocated to respective phases of the intersection to allow movement of the vehicles. One green time duration is active at time. When GT1 is active then only vehicles of phase 1 are moving and vehicles of phase 2 are stopped (i.e. red light signal for phase 2). Similarly when GT2 is active then only vehicles of phase 2 are moving and vehicles of phase 1 are stopped (i.e. red light signal for phase 1).

1. If (TQ1 is VS) and (TQ2 is VS) then (CycleLength is S)

2. If (TQ1 is VS) and (TQ2 is S) then (CycleLength is M)

3. If (TQ1 is VS) and (TQ2 is M) then (CycleLength is M)

4. If (TQ1 is VS) and (TQ2 is L) then (CycleLength is L)

5. If (TQ1 is VS) and (TQ2 is VL) then (CycleLeng th is VL)

6. If (TQ1 is $\mathrm{S}$ ) and (TQ2 is $\mathrm{S}$ ) then (CycleLeng th is M)

7. If (TQ1 is S) and (TQ2 is M) then (CycleLength is L)

8. If (TQ1 is S) and (TQ2 is L) then (CycleLength is L)

9. If (TQ1 is S) and (TQ2 is VL) then (CycleLength is VL)

10. If (TQ1 is M) and (TQ2 is M) then (CycleLeng th is L)

11. If (TQ1 is M) and (TQ2 is $\mathrm{L}$ ) then (CycleLength is VL)

12. If (TQ1 is M) and (TQ2 is VL) then (CycleLeng th is VL)

13. If (TQ1 is L) and (TQ2 is L) then (CycleLength is VL)

14. If (TQ1 is L) and (TQ2 is VL) then (CycleLength is VL)

15. If (TQ1 is VL) and (TQ2 is VL) then (CycleLength is VL)

16. If (TQ1 is $\mathrm{S}$ ) and (TQ2 is VS) then (CycleLength is $\mathrm{M}$ )

17. If (TQ1 is $\mathrm{M})$ and (TQ2 is VS) then (CycleLength is M)

18. If (TQ1 is M) and (TQ2 is S) then (CycleLength is $\mathrm{L}$ )

19. If (TQ1 is $L$ ) and (TQ2 is VS) then (CycleL ength is L)

20. If (TQ1 is L) and (TQ2 is S) then (CycleLength is L)

21. If (TQ1 is $\mathrm{L}$ ) and (TQ2 is M) then (CycleLength is VL)

22. If (TQ1 is VL) and (TQ2 is VS) then (CycleLength is VL)

23. If (TQ1 is VL) and (TQ2 is S) then (CycleLength is VL)

24. If (TQ1 is VL) and (TQ2 is M) then (CycleLeng th is VL)

25. If (TQ1 is VL) and (TQ2 is L) then (CycleLength is VL)

Fig. 3 Fuzzy rules of FVCS

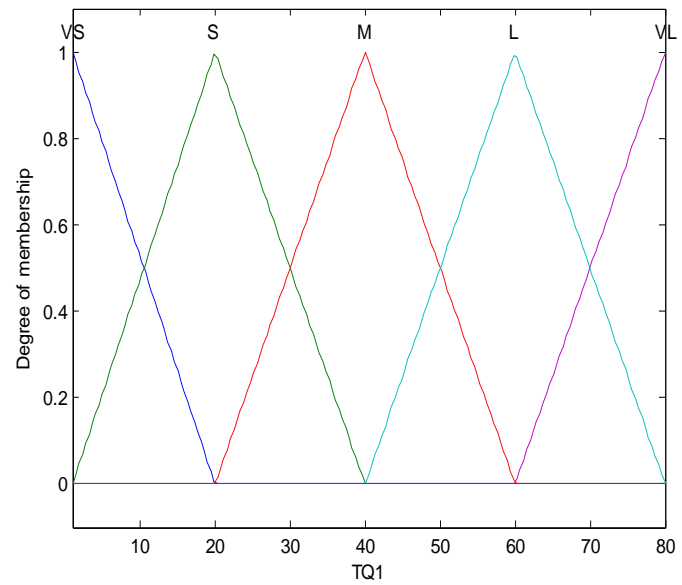

Fig. 4 Triangular Member function of TQ1

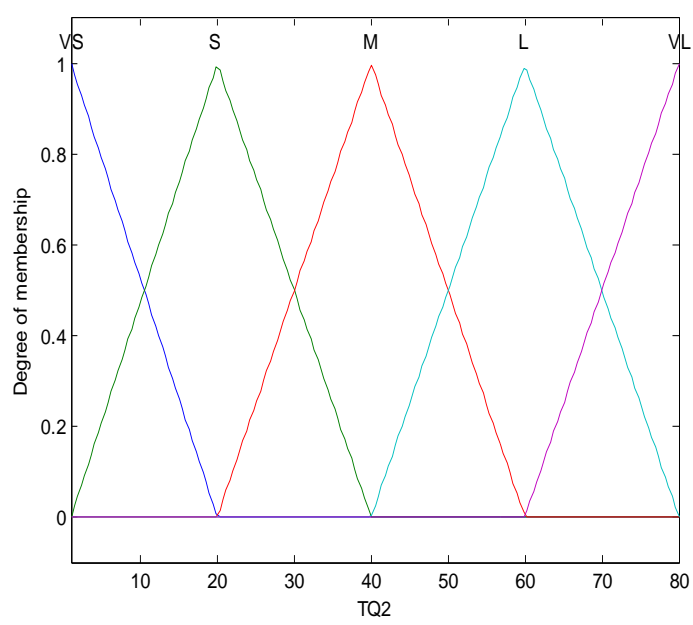

Fig. 5 Triangular Member function of TQ2

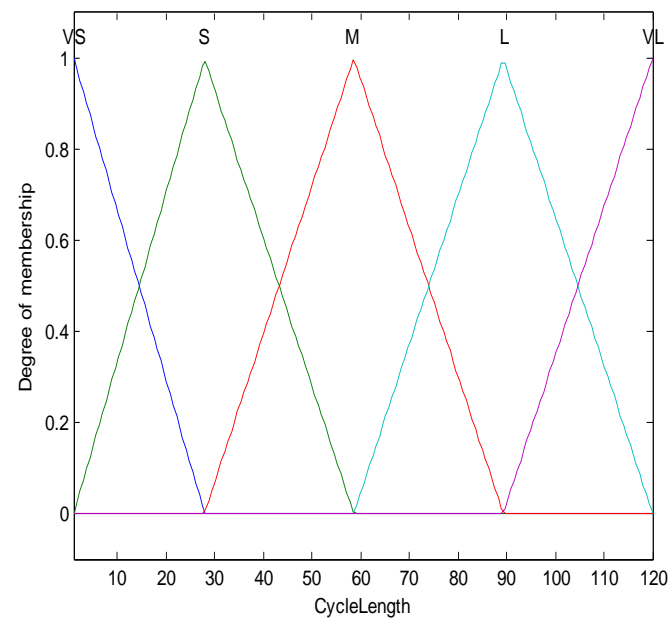

Fig. 6 Triangular Member function of Cycle Length

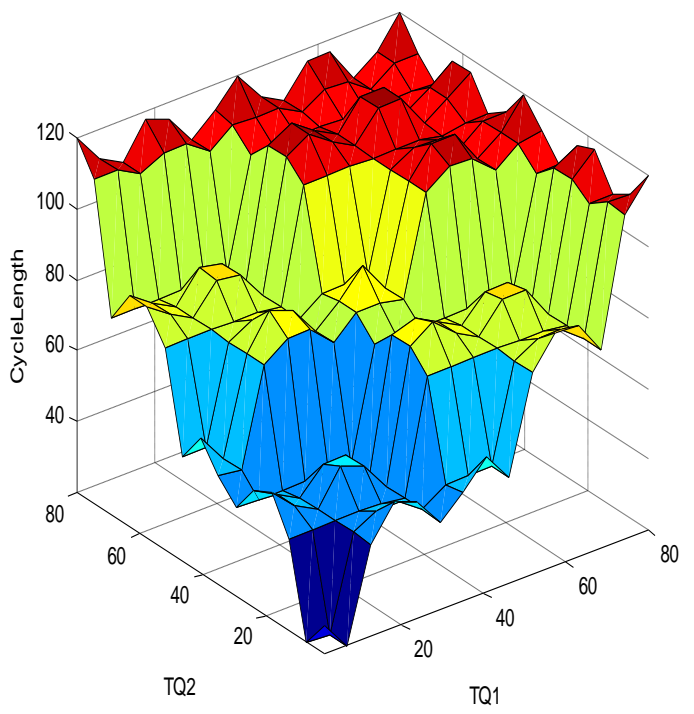

Fig. 7 Surface Area of FIS 


\section{EXPERIMENT}

Experiments have been performed on proposed architecture with the help of MATLAB. Eight different sets of data have been taken for the experiments. Data set has various combination of number of vehicles of TQ1 and TQ2. Table I shows the data set with corresponding cycle length which has been obtained from FVCS system.

Table I Eight data set with Cycle length from FVCS

\begin{tabular}{|c|c|c|c|}
\hline $\begin{array}{l}\text { Data } \\
\text { Set }\end{array}$ & TQ1 & TQ2 & $\begin{array}{l}\text { Cycle Length } \\
\text { (Seconds })\end{array}$ \\
\hline 1 & 5 & 80 & 114.05 \\
\hline 2 & 10 & 70 & 74.78 \\
\hline 3 & 66 & 20 & 80.73 \\
\hline 4 & 40 & 50 & 74.78 \\
\hline 5 & 25 & 35 & 81.92 \\
\hline 6 & 55 & 15 & 81.92 \\
\hline 7 & 75 & 40 & 112.86 \\
\hline 8 & 61 & 20 & 87.87 \\
\hline
\end{tabular}

The results obtained from the experiments have been compared with traffic system with fixed cycle length. Here, we take fixed cycle length is of 60 seconds which is generally used in many intersections. A survey has been conducted regarding selection of fixed cycle length. Table II is showing data set with fixed cycle length. In the case of fixed cycle length of 60 seconds, half of the cycle length i.e. 30 seconds is allotted to green time duration for each phase.

Table II Eight data set with fixed cycle length

\begin{tabular}{|c|c|c|c|}
\hline $\begin{array}{l}\text { Data } \\
\text { Set }\end{array}$ & TQ1 & TQ2 & $\begin{array}{l}\text { Cycle Length } \\
\text { Fixed Time } \\
\text { (Seconds) }\end{array}$ \\
\hline 1 & 5 & 80 & 60 \\
\hline 2 & 10 & 70 & 60 \\
\hline 3 & 66 & 20 & 60 \\
\hline 4 & 40 & 50 & 60 \\
\hline 5 & 25 & 35 & 60 \\
\hline 6 & 55 & 15 & 60 \\
\hline 7 & 75 & 40 & 60 \\
\hline 8 & 61 & 20 & 60 \\
\hline
\end{tabular}

Fig. $8 \& 9$ are showing fixed time cycle length scenario with respect to phase 1 and phase 2 . Horizontal axis is showing number of data set and vertical axis is showing number of vehicles in Fig. $8 \&$ 9. Both figures are showing number of vehicles (i.e. TQ) presents on a phase (TQ1 for phase 1 and TQ2 for phase 2), number of vehicles that have crossed the intersection and number of vehicles which are waiting at intersection for crossing it.

Both Fig. 8 \& 9 indicate that numbers of waiting vehicles are large in the fixed cycle length vehicle control system. If there are many vehicles present at phases then there are more number of vehicles are in the queue which are waiting for their turn to cross the intersection in the fixed cycle length vehicle control system.

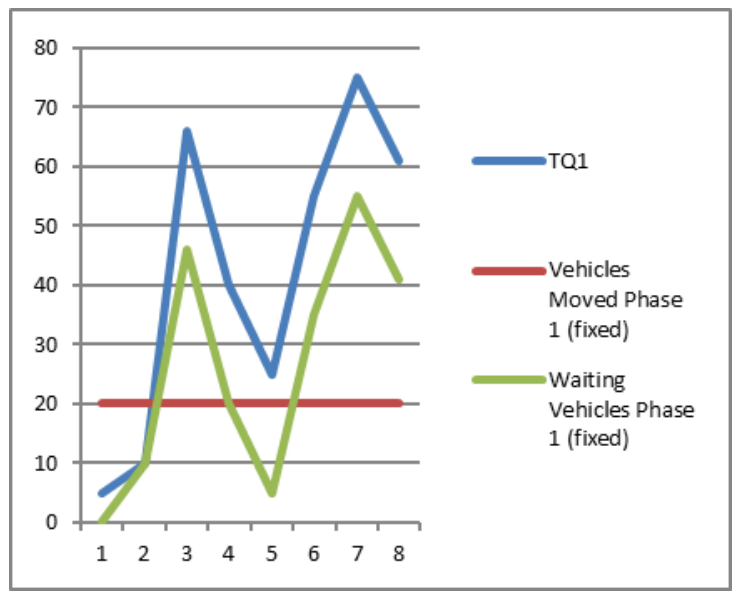

Fig. 8 Fixed Time Cycle Length (Phase 1)

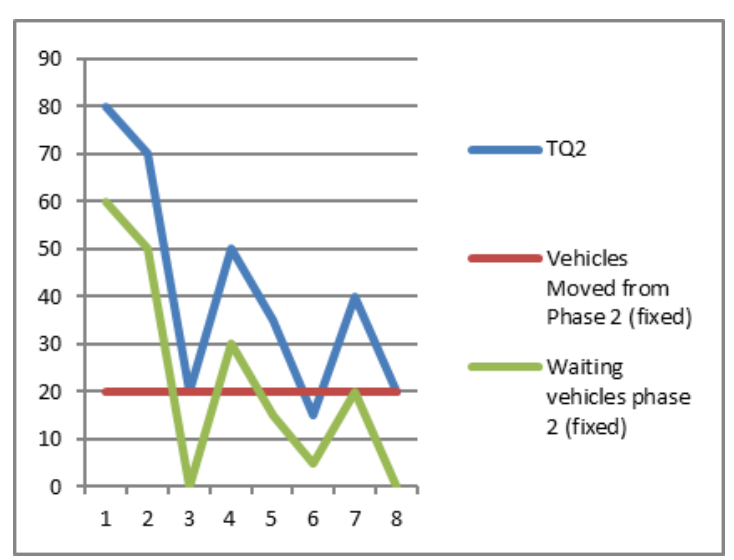

Fig. 9 Fixed Time Cycle Length (Phase 2)

Fig. $10 \& 11$ are showing effects of dynamic allocation of cycle lengths that are provided by FVCS. Horizontal axis is showing number of data set and vertical axis is showing number of vehicles in Fig. 10 \& 11. FVCS provides suitable cycle length after observing the current traffic of both the phases. Both Fig. $10 \& 11$ are also showing that number of waiting vehicles are very less as compared to fixed cycle length traffic system. Fig. $10 \& 11$ consist of number of vehicles presently available on a phase (TQ1 for phase 1 and TQ2 for phase 2), number of vehicles that have moved through the intersection in a phase and number of waiting vehicles in a phase. 


\section{RESULT}

This section discusses the result that has been obtained from the experiment. The experiment has been carried out using MATLAB. Fig. 12 shows the result of the experiment. It shows a reduction of $30.56 \%$ in number of waiting vehciles in phase 1 and a reduction of $27.27 \%$ in number of waiting vehciles in phase 2. An avegare of $28.92 \%$ reduction in number of waiting vehicles because of proposed FVCS architecture.

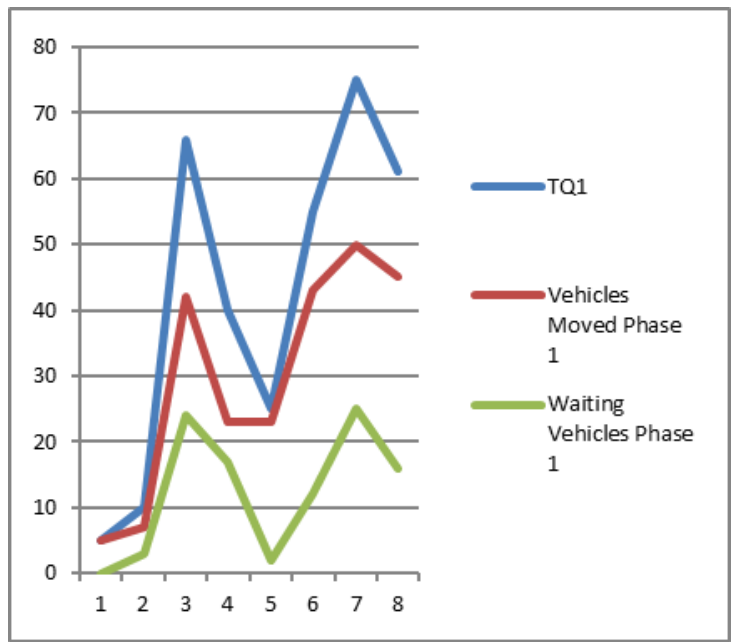

Fig. 10 FVCS Time Cycle Length (Phase 1)

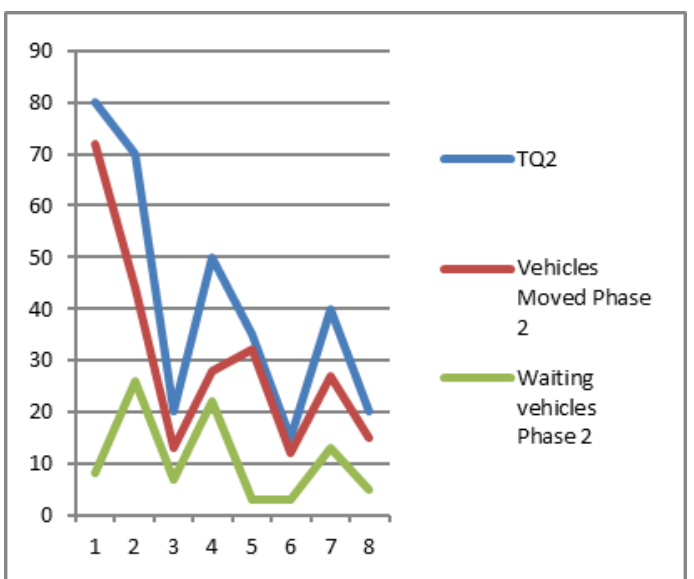

Fig. 11 FVCS Time Cycle Length (Phase 2)

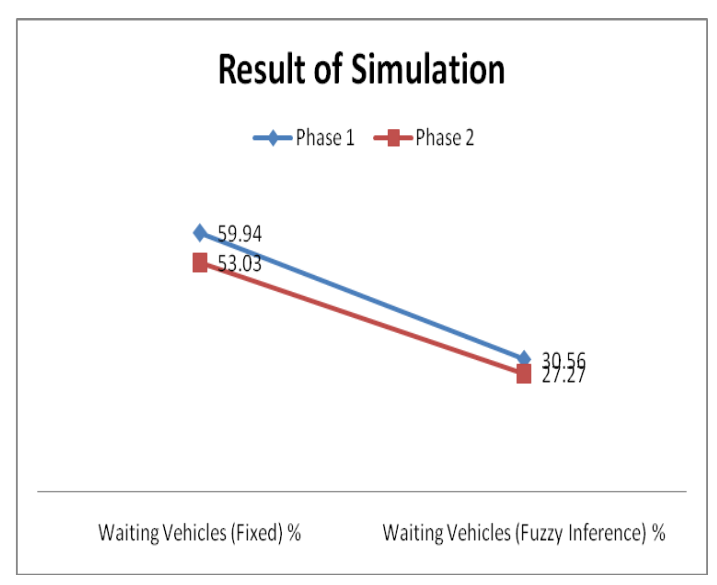

Fig. 12 Result of Experiment

\section{CONCLUSION}

Traffic jam is major problem at the intersection in the urban cities. Fixed signal timings traffic system is not effective to handle current traffic jam. Proposed architecture FVCS using fuzzy logic shows its effectiveness to handle current dynamic traffic. FVCS is able to handle the dynamic pattern of incoming vehicles by providing dynamic cycle length at the intersection. FVCS reduces number of vehicles on an average of $28.92 \%$ that are waiting at the intersection to cross the intersection. The work can be extended to handle vehicles at multiple intersections which are adjacent to each other.

\section{REFERENCES}

1. Graham I, Jones PL. "Expert systems: knowledge, uncertainty, and decision", Chapman \& Hall, Ltd.; 1, pp. 117-118, (1988).

2. Hegyi A, De Schutter B, Hoogendoorn S, Babuska R, Van Zuylen H, Schuurman H., "A fuzzy decision support system for traffic control centers", Intelligent Transportation Systems, pp. 358-363. IEEE (2001)

3. C. Taylor, D. Meldrum, "Fuzzy Ramp Implementation", Research Project prepared by Department of Electrical Engineering of University of Washington, at Seattle, Washington 98195, pp. 1, 6, (2000).

4. Day, Scoot Split, "Cycle \& Offset Optimization Technique", presented by Siemens AG, Traffic Control Systems Division, presented to Transportation Research Board, TRB Committee A3A18 Traffic Signal Systems, TRB Mid- Year Meeting and Adaptive Traffic Signal Control Workshop, pp. 6,9,12,13 (1998)

5. NIITTYMaki, J., and Marko Mäenpää. "Fuzzy public transport priority in traffic signal control." In Transport Systems Organisation And Planning. Proceedings Of The 3rd KFB Research Conference, Held In Stockholm, Sweden, 13th To 14th June 2000.

6. Wei, Wu, and Mingjun Wang. "Traffic signal control using fuzzy and neural network." In 8th International Conference on Neural Information Processing, Shanghai, China. 2001.

7. Liu, H.X., Ma, W., Hu, H., Wu, X. and Yu, G., "SMART-SIGNAL: Systematic Monitoring of Arterial Road Traffic Signals", In: 11th International IEEE Conference on Intelligent Transportation Systems, Beijing, pp. 1061-1067 (2008).

8. Kulkarni, G.H. and Waingankar, P.G, "Fuzzy Logic Based Traffic Light Controller", In: Second International Conference on Industrial and Information Systems, pp. 107-110 (2007).

9. Wang, Q., Wang, L. and Guangcun, W., "Research on Traffic Light Adjustment Based on Compatibility Graph of Traffic", In: Third International Conference on Intelligent Human-Machine Systems and Cybernetics, pp. 88-92 (2011).

10. Zhang, Lin, Honglong Li, and Panos D. Prevedouros. "Signal control for oversaturated intersections using fuzzy logic." In Transportation Research Board Annual Meeting, Washington DC, USA. 2005.

11. Pappis, Costas P., and Ebrahim H. Mamdani. "A fuzzy logic controller for a trafc junction." IEEE Transactions on Systems, Man, and Cybernetics 7, no. 10 (1977): 707-717.

12. L. A. Akanbi and E. A. Olajubu., "A fuzzy-Based Intelligent Traffic Control System for Managing VIP-Induced Chaos at Road Intersections", African Journal of Computing \& ICT, Vol 5. No. 4, June, (2012).

13. Leung, Ricky WK, Henry CW Lau, and C. K. Kwong. "On a responsive replenishment system: a fuzzy logic approach." Expert Systems 20, no. 1 (2003): 20-32

14. Hamed Homaei, S. R. Hejazi, Seyed Ali Mohamad Dehghan, "A New Traffic Light Controller Using Fuzzy Logic for a Full Single Junction Involving Emergency Vehicle Preemption", Journal of Uncertain Systems Vol.9, No.1, pp.49-61, (2015)

15. Asaithambi, Gowri, Venkatesan Kanagaraj, and Tomer Toledo. "Driving behaviors: Models and challenges for non-lane based mixed traffic." Transportation in Developing Economies 2, no. 2 (2016): 19.

16. Riaz, F. \& Niazi, M.A., "Road collisions avoidance using vehicular cyber-physical systems: a taxonomy and review", Complex Adaptive Systems Modeling 4: 15. doi:10.1186/s40294-016-0025-8 (2016).

17. Jose E. Naranjo, Miguel A. Sotelo, Carlos Gonzalez, Ricardo Garcia, Teresa De Pedro, "Using Fuzzy Logic in Automated Vehicle Control", IEEE Intelligent Systems, Volume: 22, Issue: 1, Jan 2007 pp $36-45$ (2007)

\section{Published By:}

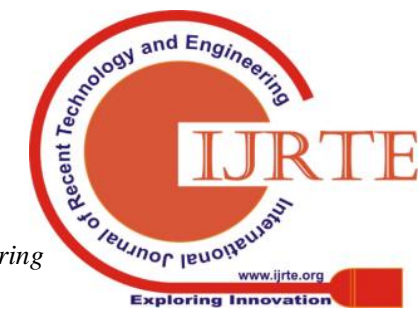


18. Yin, He, Wenhao Zhou, Mian Li, Chengbin Ma, and Chen Zhao. "An adaptive fuzzy logic-based energy management strategy on battery/ultracapacitor hybrid electric vehicles." IEEE Transactions on Transportation Electrification 2, no. 3 (2016): 300-311.

19. Kisacikoglu, M. C., M. Uzunoglu, and M. S. Alam. "Fuzzy logic control of a fuel cell/battery/ultra-capacitor hybrid vehicular power system." In 2007 IEEE vehicle power and propulsion conference, pp. 591-596. IEEE, 2007.

20. Sordi Filho, Álvaro Luiz, Leonardo Presoto de Oliveira, André Schneider de Oliveira, João Alberto Fabro, and Marco Aurélio Wehrmeister. "Simultaneous Navigation and Mapping in an Autonomous Vehicle Based on Fuzzy Logic." Designing with Computational Intelligence, pp. 53-68. Springer, Cham, 2017.

21. Shiri, MJ Shirvani, and Hamid Reza Maleki. "Maximum Green Time Settings for Traffic-Actuated Signal Control at Isolated Intersections Using Fuzzy Logic." International Journal of Fuzzy Systems 19, no. 1 (2017): 247-256

22. Luo, Quyuan, Xuelian Cai, Tom H. Luan, and Qiang Ye. "Fuzzy logic-based integrity-oriented file transfer for highway vehicular communications." EURASIP Journal on Wireless Communications and Networking 2018, no. 1 (2018): 3.

\section{AUTHORS PROFILE}

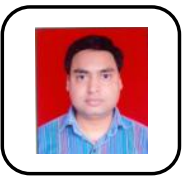

Mr. Shashank Sahu received his B.E. in Compute from North Maharashtra University, Jalgaon, India in 1996. He received his M.Tech degree in Computer Science \& Engineering from Gautam Buddh Technical University, Lucknow, India, in 2010. He is pursuing Ph.D in Computer Science \& Engineering from Sharda University, Greater Noida, India. He is working as Associate Professor in Department of Computer Science \& Engineering at Ajay Kumar Garg Engineering College Ghaziabad, India. He has 22 years academic experience. He has guided $12 \mathrm{M}$.Tech (CSE) students and several B.Tech (CSE) projects. He has published more than 35 research papers in various International/National Journals and Conferences. His research areas are Software Engineering, Web Technologies, Evolving Agents and Programming Paradigm.

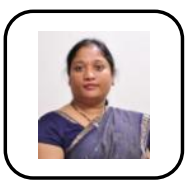

Dr. Rashi Agarwal is at present Professor in the Department of Computer Applications in Galgotias College of Engineering and Technology, Greater Noida, India. Dr. Rashi did Ph.D. (Computer Science) from Gautum Budth Technical University, Lucknow, India in 2011. Her area of research is Fractal Geometry and its applications in computer science, in which she has been guiding M.Tech. and Ph.D. students. She has published number of research papers in International Journals and Conferences. Her publication is focused on stability of fractals under different type of perturbations. She has 18 year of experience in teaching graduate and post graduate students.

Dr. Rajesh Kumar Tyagi, after receiving M.Sc degree in Physics (Electronics) from CCS University Meerut, Uttar Pradesh and M.Tech degree in Computer Science and Engineering from Kurukshetra University, Haryana, India, completed his Ph.D. degree from University School of Engineering and Technology, Guru Gobind Singh Indraprastha University, New Delhi, India. He is working as Professor in Department of Computer Science \& Engineering at Amity University, Gurugram, India. Dr. Tyagi has vast teaching and research experience. He has worked with Amity School of Engineering and Technology (ASET) Delhi and served various other reputed Organizations as Professor such as Krishna Institute of Engineering and Technology (KIET) and SRM University, NCR Campus and has published more than 40 research papers in various reputed International/National Journals/Conferences. He has guided several M.Tech (CSE) and B.Tech (CSE/IT) projects and is also currently guiding no. of Ph.D. students. He is on the editorial and reviewer board of several reputed International Journals and has organized Conferences and has also delivered lectures as keynote speakers in International/ National Conferences, Seminars, FDP and Workshops. He has handled various responsibilities like Head of the Department, Chairman of Project Evaluation Committee, Head Entrepreneurship-Cell and Expert member in selection committees etc. His research areas of interest include Ad hoc Networks, Machine Learning, Cloud computing, Cryptography, Network Security and Modeling \& Simulation of Nano-Devices 